\title{
Self-focusing and self-trapping in unbiased centrosymmetric photorefractive media
}

\author{
B. Crosignani, E. DelRe, and P. Di Porto \\ Dipartimento di Fisica, Università dell'Aquila, 67010 L'Aquila, Italy, and Istituto Nazionale di Fisica della Materia, \\ Unità di Roma I, Rome, Italy

\section{A. Degasperis} \\ Dipartimento di Fisica, Università di Roma "La Sapienza," 00185 Rome, Italy, and Istituto Nazionale di Fisica Nucleare, \\ Sezione di Roma I, Rome, Italy \\ Received February 12, 1998 \\ We predict self-focusing and self-trapping of optical beams propagating in unbiased centrosymmetric \\ photorefractive crystals in the near-transition paraelectric phase, where the nonlinear response is proportional \\ to the square of the diffusion space-charge field. (C) 1998 Optical Society of America \\ OCIS codes: $190.0190,190.5330,190.5940,160.5320$.
}

In the past few years, nonlinear optical propagation in photorefractive (PR) crystals has been the object of an extensive research effort, which has eventually demonstrated the possibility of achieving self-focusing and self-trapping of optical beams. ${ }^{1}$ In particular, spatial solitons can be supported by PR crystals that exhibit a linear electro-optic effect and result from a nonuniform screening of an external bias electric field. In this process the inherently asymmetric internal spacecharge diffusion field ${ }^{1} E_{\mathrm{sc}}=-\left(K_{B} T / e\right) \nabla\left[\ln \left(I+I_{d}\right) / I_{d}\right]$, where $I$ is the optical intensity and $I_{d}$ is the dark irradiance of the crystal, plays a negligible role. Actually, it can be shown analytically ${ }^{2}$ that, in the absence of external bias, the diffusion field alone can support in such crystals one-dimensional nondiffracting planar beams (which undergo unavoidable self-bending during propagation).

Most recently, self-confinement in biased centrosymmetric PR crystals that exhibit a quadratic (but not linear) electro-optic effect was considered both theoretically ${ }^{3}$ and experimentally. ${ }^{4}$ In this case, because the nonlinear refractive-index contribution for unbiased centrosymmetric crystals is proportional to the square of $E_{\mathrm{sc}}$, its asymmetry is no longer relevant, and it is natural to look for the existence of self-confined propagating beams with no self-bending. In this Letter we study the general propagation equation in unbiased centrosymmetric PR crystals and demonstrate the existence of such solutions in the form of both selfconfined and self-focused beams.

In centrosymmetric media the photoinduced change of refractive index is given by ${ }^{3} \Delta n=-(1 / 2) n^{3} g \varepsilon_{0}^{2}\left(\varepsilon_{r}-\right.$ $1)^{2} E_{\mathrm{sc}}{ }^{2}$, where $n$ is the unperturbed refractive index of the crystal, $g$ is an effective quadratic electrooptic coefficient, and $\varepsilon_{r}$ is the low-frequency dielectric constant. This expression can be now inserted into the parabolic wave equation ${ }^{1}$

$$
\left[i \frac{\partial}{\partial z}+\frac{1}{2 k}\left(\frac{\partial^{2}}{\partial x^{2}}+\frac{\partial^{2}}{\partial y^{2}}\right)\right] A+\frac{k}{n} \Delta n A=0
$$

which describes the evolution of the slowly varying amplitude $A$ of the propagating optical field $E_{\text {opt }}=$ $A(x, z) \exp (i k z-i \omega t)+$ c.c., where $k=n \omega / c$. Proceeding in this way, and confining our attention to the one- plus one-dimensional $(1+1 \mathrm{D})$ case, we obtain

$$
\left(i \frac{\partial}{\partial \zeta}+\frac{\partial^{2}}{\partial \xi^{2}}\right) A+\gamma \frac{\left(\partial|A|^{2} / \partial \xi\right)^{2}}{|A|^{4}} A=0
$$

where $\zeta=k z, \xi=\sqrt{2} k x$, and $\gamma=-n^{2} k^{2} g \varepsilon_{0}^{2}\left(\varepsilon_{r}-\right.$ $1)^{2}\left(K_{B} T / e\right)^{2}$. This model equation applies if we neglect the dark irradiance $I_{d}$ with respect to the intensity $I=|A|^{2}$, a condition that obviously requires the peak intensity $I_{0}$ to be much larger than $I_{d}$. We note that, because of the structure of Eq. (2), we can always account for the influence of the loss term $i \alpha A$, which has been omitted, by multiplying its solutions by $\exp (-\alpha \zeta)$. Moreover, if $A(\xi, \zeta)$ is a solution, then $a A\left[p\left(\xi-\xi_{0}\right), p^{2}\left(\zeta-\zeta_{0}\right)\right]$ solves Eq. (2) for arbitrary real $p, \xi_{0}$, and $\zeta_{0}$ and complex $a$. This property, which is valid as far as the relative scaling between longitudinal and transverse coordinates is concerned and also if $I_{d}$ is not neglected, implies that localized solutions that correspond to self-guided propagation (when they exist; see below) cannot possibly obey a peak amplitudewidth relation (the so-called existence curve) as in the case of Kerr-type or ordinary photorefractive solitons.

To solve Eq. (2) it is expedient to introduce the new independent variable $B$ through the transformation $A=B^{\mu}$, where $\mu=1 /(1+4 \gamma)$. Different types of solution of Eq. (2) can then be associated with the sign of the parameter $\mu$. Let us first consider the case $\mu>0$, i.e., $\gamma>-1 / 4$. A particular solution of Eq. (2) reads as

$$
\begin{aligned}
A(\xi, \zeta)= & \frac{A_{0} \exp [i \phi(\xi, \zeta)]}{\left(1+16 \zeta^{2} / \mu \delta^{4}\right)^{1 / 4}} \exp \left[-\frac{\xi^{2}}{\delta^{2}\left(1+16 \zeta^{2} / \mu \delta^{4}\right)}\right] \\
& \times\left\{H_{n}\left[(2 / \mu)^{1 / 2} \frac{\xi}{\delta\left(1+16 \zeta^{2} / \mu \delta^{4}\right)^{1 / 2}}\right]\right\}^{\mu}, \quad \text { (3) }
\end{aligned}
$$


where $A_{0}$ and $\delta$ are arbitrary parameters fixed by the $\zeta=0$ boundary beam profile, $H_{n}$ are Hermite polynomials, and

$$
\begin{aligned}
\phi(\xi, \zeta)= & \frac{4 \zeta \xi^{2}}{\mu \delta^{4}\left(1+16 \zeta^{2} / \mu \delta^{4}\right)}-\mu^{1 / 2} \\
& \times(n+1 / 2) \arctan \left(4 \zeta / \mu^{1 / 2} \delta^{2}\right) .
\end{aligned}
$$

In the limiting case $\mu \rightarrow \infty$, that is, for $\gamma=-1 / 4$, for all even values of $n$ there is no diffraction because these solutions all take the same Gaussian expression:

$$
\begin{aligned}
A(\xi, \zeta)= & A_{0} \exp \left[-2 i(1+2 n) \zeta / \delta^{2}\right] \\
& \times \exp \left[-(1+2 n) \xi^{2} / \delta^{2}\right]
\end{aligned}
$$

From an experimental point of view, the most relevant solution of the kind given in Eq. (3) corresponds to $n=0$ (i.e., $H_{0}=1$ ); in fact, in this case the boundary condition has a Gaussian form and can easily be imposed. Referring to this situation, it is important to note that $\mu$ larger than unity (i.e., $-1 / 4<\gamma<0$ ) gives rise to nonlinear self-focusing, whereas $\mu$ smaller than unity (i.e., $\gamma>0$ ) leads to defocusing with respect to linear diffraction corresponding to $\mu=1(\gamma=0)$. For the special value $\gamma=-1 / 4$ the solution has the expression given by Eq. (5) with $n=0$.

For comparison, we note that solutions analogous to those found in Ref. 2 for noncentrosymmetric crystals exist also in our case and read as

$$
\begin{aligned}
A(\xi, \zeta)= & A_{0} \exp \left\{-i\left[\eta \zeta\left(\xi+2 \eta \zeta^{2} / 3\right)\right]\right\} \\
& \times\left\{\operatorname{Ai}\left[(\eta / \mu)^{1 / 3}\left(\xi+\eta \zeta^{2}\right)\right]\right\}^{\mu},
\end{aligned}
$$

where $A i(x)$ is the Airy function and $\eta$ is a free parameter that is related to its main width.

Let us now consider the case $\mu<0$ (i.e., $\gamma<-1 / 4$ ). In this case, particular solutions in the form of solitary waves are

$$
\begin{aligned}
A(\xi, \zeta)= & A_{0} \exp [i(\chi \xi-\beta \zeta)] \\
& \times\left\{\frac{1}{\cosh \left[(\beta /|\mu|)^{1 / 2}(\xi-2 \chi \zeta)\right]}\right\}^{|\mu|},
\end{aligned}
$$

where $\chi$ is a constant that depends on the phase modulation at the boundary $\zeta=0$ and is responsible for the beam's transverse displacement at a constant rate $2 \chi$, and $\beta$ relates to signal width. A class of solutions can also be obtained from Eq. (3) through the substitution $\mu \rightarrow-\mu$. However, because of the negative value of $\mu$, the Hermite polynomials in Eq. (3) now appear in the denominator and, therefore, their zeros are singularities in the variable $\xi$ of our solution. This result implies that the only physically acceptable solutions obtain for even-degree polynomials, i.e., for $n=2 \mathrm{~m}$. For instance, the solution associated with $H_{0}$ is

$$
\begin{aligned}
A(\xi, \zeta)= & \frac{A_{0} \exp [-i \phi(\xi, \zeta)]}{\left(1-16 \zeta^{2} /|\mu| \delta^{4}\right)^{1 / 4}} \\
& \times \exp \left[-\xi^{2} / \delta^{2}\left(1-16 \zeta^{2} /|\mu| \delta^{4}\right)\right],
\end{aligned}
$$

where

$$
\begin{aligned}
\phi(\xi, \zeta)= & {\left[\frac{4 \zeta \xi^{2}}{|\mu| \delta^{4}\left(1-16 \zeta^{2} /|\mu| \delta^{4}\right)}\right.} \\
& \left.+\frac{|\mu|^{1 / 2}}{4} \ln \frac{\delta^{2}|\mu|^{1 / 2}+4 \zeta}{\delta^{2}|\mu|^{1 / 2}-4 \zeta}\right] .
\end{aligned}
$$

This solution corresponds to a self-focusing process leading to catastrophic collapse unless the propagation distance is limited to values such that $\zeta<\delta^{2} \sqrt{|\mu|} / 4$.

We remind the reader that the possibility of explicitly finding the above analytical solutions is based on neglecting the dark irradiance $I_{d}$ (as, for example, was done in Ref. 2). If $I_{d}$ is not neglected, it is possible to show that, strictly speaking, no localized solitary wave solutions exist. However, for the large values of $I_{0} / I_{d}$ typically present in most experimental situations, our solutions are by all practical means indistinguishable from the ones that pertain to the exact model over the transverse width of the crystal and for typical crystal lengths.

Let us now discuss the possibility of experimentally observing the nonlinear propagation effects predicted by the above analytical results. In a standard experimental configuration, a polarized argon-ion laser beam operating at, for example, $\lambda=\omega / c=515 \mathrm{~nm}$ is focused by a cylindrical lens onto the input face of a zero-cut sample so as to propagate parallel to it. The beam is polarized in a plane orthogonal to the lens axis (along the confined direction). To evaluate the feasibility of observing self-focusing and eventually self-trapping we have to evaluate the order of magnitude of $\Delta n$ [see Eq. (1)], which has to be large enough to provide beam guidance. In our case the nonlinear index modulation is determined, for a given crystal temperature $T$, only by the size (and eventually the shape) of the input beam. No external parameters, such as intensity ratio (as long as $I_{0} \gg I_{b}$ ) and external voltage, are present. In particular, the index modulation, for a fixed $T$, is given by $\Delta n=|\gamma| n /\left(2 k^{2}\right)[\mathrm{d}(\ln I) / \mathrm{d} x]^{2}=|\gamma| n /\left(2 k^{2}\right) 4 x^{2} / w^{4}$, where we have assumed that $I=I_{0} \exp \left(-x^{2} / w^{4}\right)$ [see Eq. (5)]. The index change between $x=0$ and $x=2 w$ thus gives rise to an effective $\Delta n=16|\gamma| n / 2 k^{2} w^{2}$. For the special value $|\gamma|=1 / 4$ we have, for $w=6 \mu \mathrm{m}$ (corresponding to a $10-\mu \mathrm{m}$ intensity full width at half-maximum), $\Delta n=1.6 \times 10^{-4}$, which is in the range of values able to provide linear waveguiding. We stress the fact that no existence curve is present; rather, the crystal parameters have to be such as to produce the appropriate values of $\gamma$. Considering typical parameter values, such as $n \cong 2.4$, $g \cong 0.15 \mathrm{~m}^{4} \mathrm{C}^{-2}$, and $\left(K_{B} T / e\right) \cong 26 \mathrm{mV}$ at room temperature, we need high values of $\varepsilon_{r}$ to reach the necessary order of magnitude. Such values are attainable only in paraelectric materials close to the ferroelectric phase transition. For example, samples of strontium barium niobate ${ }^{5}$ can have values of $\varepsilon_{r} \cong$ $2 \times 10^{4}-8 \times 10^{4}$ for $T \cong 125-140^{\circ} \mathrm{C}$, and we expect to be able to observe significant diffusion-driven nonlinear diffraction compensation (the effective $\Delta n$ 
ranges in this case from $1.8 \times 10^{-5}$ to $2.7 \times 10^{-4}$ ). Other crystals with high $\varepsilon_{r}$ are paraelectric potassium lithium tantalate (KTN $)^{6}$ and paraelectric potassium lithium tantalate niobate (KLTN). ${ }^{7}$

Returning to Eq. (2), we find that the predicted nonlinear behavior depends on the light-input conditions and the value of the parameter $\mu$. For the above-mentioned configuration the input beam is an unchirped focused one-dimensional Gaussian beam. For the case of paraelectric strontium barium niobate, $\gamma$ takes values of approximately -0.03 to -0.5 , so positive values of $\mu$ larger than approximately 1.1 and negative values less than -1 are possible. This means that, in principle, self-focusing described by Eq. (3) (with $n=0$ ), Gaussian solitons described by Eq. (5) (again with $n=0$ ), and hyperbolic solitons described by Eq. (7) (with $\chi=0$ ) can be observed.

As far as paraelectric KLTN is concerned, recent results for spatial screening solitons ${ }^{4}$ were obtained for crystals characterized by a room-temperature phase transition that, however, possesses low peak values of $\varepsilon_{r}\left(\varepsilon_{r} \approx 3 \times 10^{4}\right)$. This implies that we can explore values for at most $\mu \approx 1-1.2$ and that, therefore, we can expect only slight self-focusing effects described by Eq. (3). In this respect, we note that KLTN crystals with higher peak values of $\varepsilon_{r}$ have been grown ${ }^{7}$ but with critical behavior well below room temperature. The lower peak value of $\varepsilon_{r}$ at the phase transition has been attributed to a less homogeneous distribution of dopant and impurity concentrations. Thus it should be anticipated that for localized areas of particular samples the actual value of $\varepsilon_{r}$ will be much higher than the average bulk measured value; therefore, for welllocalized beams in short crystals, higher values of $\mu$ should be attainable.

Finally, we note that the analytical solutions can also be found easily in the $(1+2 \mathrm{D})$ case, as long as the nonlinear propagation can be described by a scalar parabolic equation, which implies some restrictions on the polarization state of the input beam and on the values of the components of the crystal electro-optic tensor.

Useful discussions with M. Segev are gratefully acknowledged.

\section{References}

1. For an up-to-date bibliography see, e.g., B. Crosignani, P. Di Porto, A. Degasperis, M. Segev, and S. Trillo, J. Opt. Soc. Am. B 14, 3078 (1997).

2. D. N. Christodoulides and T. H. Coskun, Opt. Lett. 21, 1460 (1996).

3. M. Segev and A. Agranat, Opt. Lett. 22, 1299 (1997).

4. E. DelRe, B. Crosignani, M. Tamburrini, M. Segev, M. Mitchell, E. Refaeli, and A. Agranat, Opt. Lett. 23, 421 (1998).

5. Y. Xu, Ferroelectric Materials and Their Applications (North-Holland, Amsterdam, 1991).

6. A. Agranat, V. Leyva, and A. Yariv, Opt. Lett. 14, 1017 (1989).

7. A. Agranat, R. Hofmeister, and A. Yariv, Opt. Lett. 17, 713 (1992). 